\title{
Circuit Implementation, Synchronization of Multistability, and Image Encryption of a Four-Wing Memristive Chaotic System
}

\author{
Guangya Peng $(\mathbb{D}$, Fuhong Min $(\mathbb{D}$, and Enrong Wang \\ School of Electrical and Automatic Engineering, Nanjing Normal University, Nanjing 210042, China \\ Correspondence should be addressed to Fuhong Min; minfuhong@njnu.edu.cn
}

Received 18 July 2017; Revised 22 October 2017; Accepted 28 November 2017; Published 1 February 2018

Academic Editor: Ephraim Suhir

Copyright (C) 2018 Guangya Peng et al. This is an open access article distributed under the Creative Commons Attribution License, which permits unrestricted use, distribution, and reproduction in any medium, provided the original work is properly cited.

\begin{abstract}
The four-wing memristive chaotic system used in synchronization is applied to secure communication which can increase the difficulty of deciphering effectively and enhance the security of information. In this paper, a novel four-wing memristive chaotic system with an active cubic flux-controlled memristor is proposed based on a Lorenz-like circuit. Dynamical behaviors of the memristive system are illustrated in terms of Lyapunov exponents, bifurcation diagrams, coexistence Poincaré maps, coexistence phase diagrams, and attraction basins. Besides, the modular equivalent circuit of four-wing memristive system is designed and the corresponding results are observed to verify its accuracy and rationality. A nonlinear synchronization controller with exponential function is devised to realize synchronization of the coexistence of multiple attractors, and the synchronization control scheme is applied to image encryption to improve secret key space. More interestingly, considering different influence of multistability on encryption, the appropriate key is achieved to enhance the antideciphering ability.
\end{abstract}

\section{Introduction}

The memristor has been thought to be the fourth basic element, and the concept was proposed by Professor Chua in 1971 [1]. The first memristor physical model was implemented by HP Labs in 2008 [2], which has lots of advantages [3], such as low energy consumption, small size, and high integration, so that it has been investigated in memory storage $[4,5]$, artificial neural networks $[6,7]$, chaotic circuits $[8,9]$, secure communications [10-12], and so forth.

Nowadays, an in-depth study of connecting the memristors with chaotic systems has been conducted by many scholars. Some researchers added the memristor model to Chua's circuit [13], and others combined the Lorenz system with memristors [14]. In recent years, a lot of attention has been paid to the research on the memristive chaotic circuits applied to synchronization, and the synchronization scheme of memristive chaos based on Lorenz system has been developed initially $[15,16]$. Master system and slave system can achieve chaotic synchronization under controlled constraints, and it has been widely used in secure communication and other fields $[17,18]$. However, a lot of literature with respect to the memristive chaotic system applied to secure communication only extracted its own chaotic sequence, without involving the memristive synchronization scheme $[12,19,20]$. In addition, the multiwings $[21,22]$ and multistability $[23,24]$ of memristive chaotic systems have been a hot topic. Nevertheless, there were few studies on the multiwing memristive circuit based on Lorenz system, and less literature took the fact that the multistability may produce different encryption effects into consideration. In contrast with the aforementioned literature, the multiwing memristive circuit, the synchronization control used in image encryption, and different influences of multistability will be carried out in this work.

In this paper, a novel four-wing memristive chaotic system is proposed by introducing a smooth flux-controlled memristor to Lorenz-like system. First of all, theoretical analyses are investigated by means of calculating dissipation and equilibrium point set. Secondly, the conventional dynamic analyses are carried out by Lyapunov exponents, bifurcation diagrams, Poincaré maps, phase diagrams, and 
so forth. The circuit of new system is devised by utilizing resistors, capacitors, op amps, and multipliers, and the results of circuit simulation are consistent well with those of numerical simulations. Then, a nonlinear feedback controller for synchronization of multistability is designed according to the system equations. The error curves and timing diagrams are used to illustrate the synchronization of different coexistence states indicating that the synchronization effect is effective. Finally, the synchronization scheme is applied to image encryption, so that the key space is more complicated. Taking the influence of different synchronization states on encryption into account, it is beneficial to select suitable keys and improve the antideciphering ability.

\section{Four-Wing Memristive Chaotic System}

Memristor is a basic circuit element describing the constitutive relation between charge and flux as $d \varphi(q)=M(q) d q$ or $d q(\varphi)=W(\varphi) d \varphi$, where $q, \varphi, M(q), W(\varphi)$ are charge, flux, memristance, and memductance [1]. A cubic flux-controlled memristor [25] described as $q(\varphi)=a \varphi+b \varphi^{3}, W(\varphi)=$ $d q(\varphi) / d \varphi$ will be carried out in this paper, where $a$ and $b$ are memristor parameters.

A new four-dimensional four-wing memristive chaotic system is obtained by adding a memristor with the constitutive relation of $W(\varphi)=d q(\varphi) / d \varphi$ to a Lorenz-like system deformed by classical Lorenz system [26]. The mathematical model can be described as

$$
\begin{aligned}
& \dot{x}_{1}=36 y_{1} z_{1}-\alpha x_{1}-z_{1} W\left(w_{1}\right) \\
& \dot{y}_{1}=\xi y_{1}-\beta x_{1} z_{1} \\
& \dot{z}_{1}=8 x_{1} y_{1}-\gamma z_{1} \\
& \dot{w}_{1}=x_{1}
\end{aligned}
$$

where $w_{1}=\varphi$ is magnetic flux; besides, $W\left(w_{1}\right)=a+b w_{1}^{2}$ is memductance. Both $a$ and $b$ are memristor parameters determining its characteristics, and $\alpha, \beta, \xi, \gamma$ are system parameters which determine the motion states. All the parameters are chosen as $a=-0.5, b=2.4, \alpha=15, \beta=8, \xi=1.68$, and $\gamma=15.15$. When initial conditions are set as $(0.1,0.1,0,0)$, the system in (1) produces four-wing chaotic attractors, as shown in Figure 1. The four Lyapunov exponents $\left(\mathrm{LE}_{i}\right)$ of the system in (1) are obtained by singular value decomposition as $\mathrm{LE}_{1}=$ $0.1354, \mathrm{LE}_{2}=0.0076, \mathrm{LE}_{3}=-0.2936$, and $\mathrm{LE}_{4}=-25.6091$. It is clear that $\mathrm{LE}_{1}>0, \mathrm{LE}_{2} \approx 0, \mathrm{LE}_{3}<0$, and $\mathrm{LE}_{4}<0$ indicating that the attractor is a strange attractor with chaotic characteristics. The Lyapunov dimension [27] of the new 4D four-wing memristive system is

$$
\begin{aligned}
D_{L} & =j+\frac{1}{\left|\mathrm{LE}_{j+1}\right|} \sum_{i=1}^{j} \mathrm{LE}_{i}=2+\frac{\left(\mathrm{LE}_{1}+\mathrm{LE}_{2}\right)}{\left|\mathrm{LE}_{3}\right|} \\
& =2+\frac{(0.1354+0.0076)}{|-0.2936|}=2.487 .
\end{aligned}
$$

It can be seen that the Lyapunov dimension is fractional which means it is chaotic system. The phase diagrams listed in Figure 1 show that the chaotic attractor trajectory has ergodicity and boundedness in a specific attraction domain.

Equation (5) shows the dissipation of the system in (4):

$$
\nabla V=\frac{\partial \dot{x}_{1}}{\partial x_{1}}+\frac{\partial \dot{y}_{1}}{\partial y_{1}}+\frac{\partial \dot{z}_{1}}{\partial z_{1}}+\frac{\partial \dot{w}_{1}}{\partial w_{1}}=-\alpha+\xi-\gamma
$$

when these parameters involved in (3) are selected as $\alpha=15$, $\xi=1.68$, and $\gamma=15.15$; that is, $\nabla V=-\alpha+\xi-\gamma=-15+1.68-$ $15.15=-28.47<0$. It means that system in (1) is dissipative, and the system trajectories starting from the region converge exponentially with time and form chaos asymptotically.

Let $\dot{x}_{1}=\dot{y}_{1}=\dot{z}_{1}=\dot{w}_{1}=0$; the equilibrium points in (1) are obtained as a set $A=\left\{\left(x_{1}, y_{1}, z_{1}, w_{1}\right) \mid x_{1}=y_{1}=z_{1}=\right.$ $\left.0, w_{1}=l_{1}\right\}$, where $l_{1}$ is a real constant; thus all points on the $w_{1}$-axis are equilibrium points. The Jacobi matrix $J_{A}$ of the new four-wing memristive chaotic system at the equilibrium point set is got:

$$
J_{A}=\left[\begin{array}{cccc}
-\alpha & 36 z_{1} & 36 y_{1}-W\left(w_{1}\right) & -z_{1}\left(2 b w_{1}\right) \\
-\beta z_{1} & \xi & -\beta x_{1} & 0 \\
8 y_{1} & 8 x_{1} & -\gamma & 0 \\
1 & 0 & 0 & 0
\end{array}\right] .
$$

The eigenvalue equation of the equilibrium point set $A$ is

$$
\begin{aligned}
& \lambda(\lambda+\alpha)(\lambda-\xi)(\lambda+\gamma) \\
& \quad=\lambda\left[\lambda^{3}+(\alpha+\gamma-\xi) \lambda^{2}+(\alpha \gamma-\alpha \xi-\gamma \xi) \lambda-\alpha \gamma \xi\right] \\
& \quad=0
\end{aligned}
$$

Simplify (5) as

$$
\lambda\left[\lambda^{3}+a_{1} \lambda^{2}+a_{2} \lambda+a_{3}\right]=0
$$

Equation (6) reveals that system in (1) has one zero eigenvalue and three nonzero eigenvalues, where

$$
\begin{aligned}
& a_{1}=\alpha+\gamma-\xi=28.47>0, \\
& a_{2}=\alpha \gamma-\alpha \xi-\gamma \xi=176.598>0, \\
& a_{3}=-\alpha \gamma \xi=-381.78<0 .
\end{aligned}
$$

According to the stability condition, the set of the unstable range of system in (1) can be obtained as the whole real field due to $a_{3}<0$. No matter the value of $l_{1}$, the eigenvalues of equilibrium set are calculated as $\lambda_{1}=$ $0, \lambda_{2}=-15, \lambda_{3}=1.68, \lambda_{4}=-15.15 . \lambda_{2}$ and $\lambda_{4}$ correspond to two stable solutions, and $\lambda_{3}$ is consistent with an unstable solution. Therefore, all the points on equilibrium point set are unstable. Self-excited attractors are connected with unstable equilibriums, but a hidden attractor is not connected with equilibria, for instance, those in the systems without equilibria or only one stable equilibrium [28, 29]. It is clear that the attractors in (1) are closely related to unstable equilibriums, so those are self-excited attractors. 


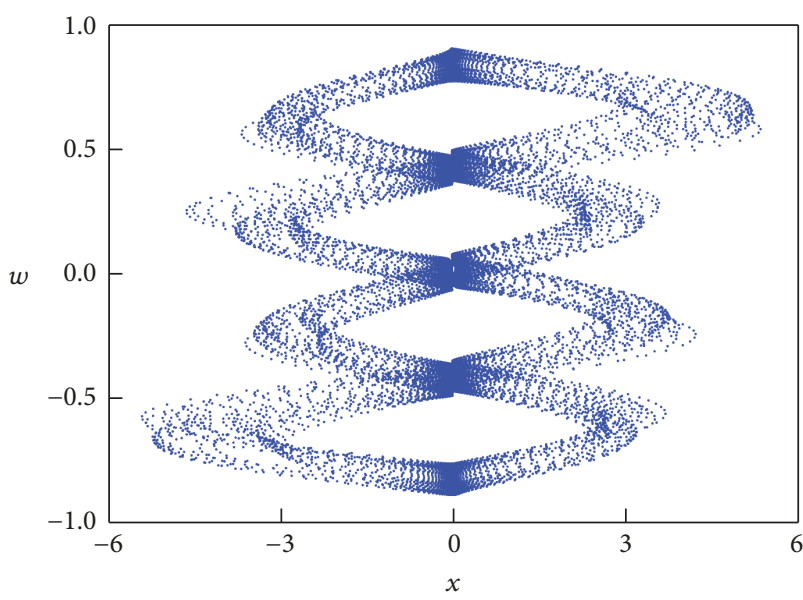

(a)

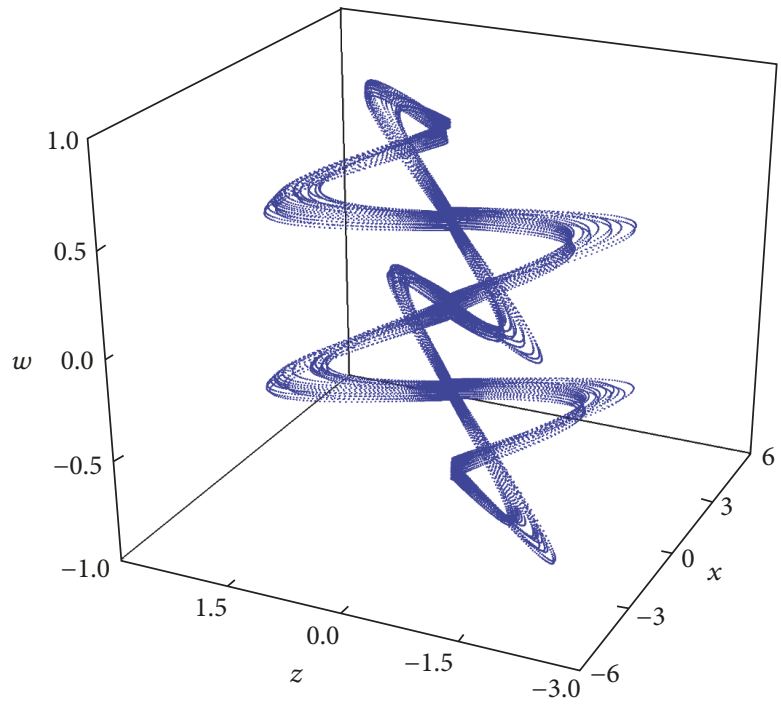

(c)

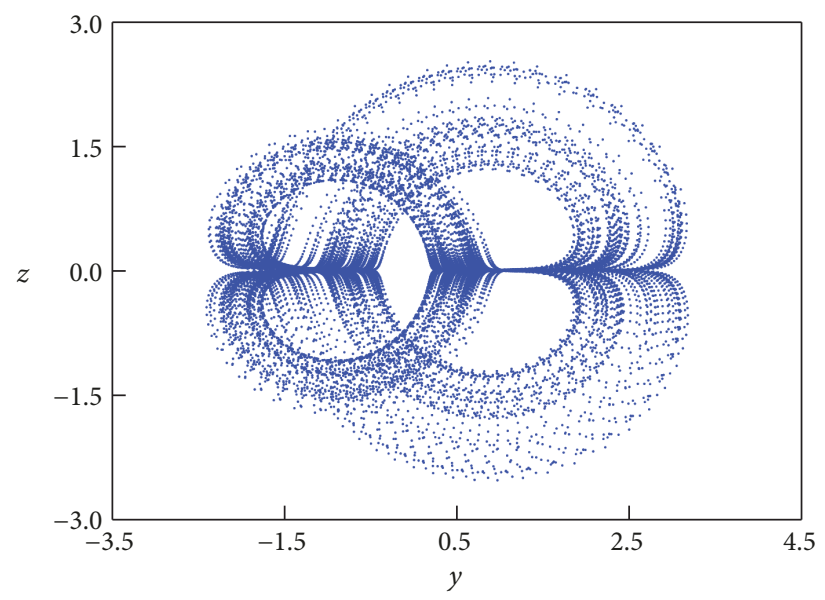

(b)

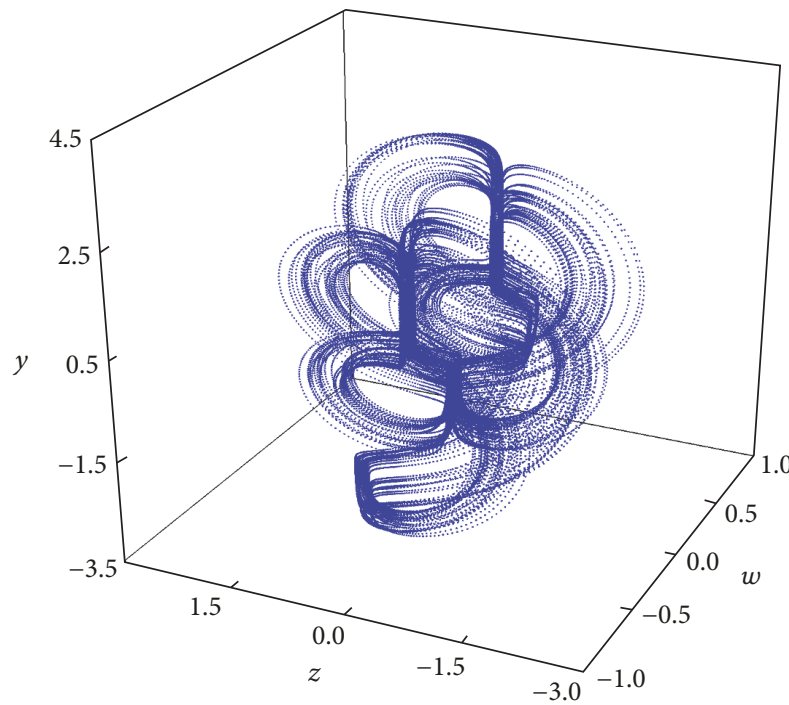

(d)

Figure 1: Phase diagrams: (a) $x-w$, (b) $y-z$, (c) $x-z-w$, and (d) $w-z-y$.

\section{Dynamic Analyses and Circuit Implementation of the New Chaotic System}

\subsection{Dynamic Analyses}

3.1.1. The Effect of System Parameter Changing. The chaotic system will present different dynamic behaviors with varying system parameters. The bifurcation diagram for the state variable $y(t)$ and the Lyapunov exponents are presented in Figure 2 with the change of the system parameter $\xi$. The initial values $(0.1,0.1,0,0)$ in (1) are taken as initial conditions, and the range of $\xi$ is $0<\xi<4.8$. The dynamics of system in (1) can be intuitively observed from Figure 2. It is clear that this system is periodic with $\xi$ varying in interval of $(1.43,1.46),(1.77,3.41)$, and $(3.50,3.95)$. The partial bifurcation diagram in Figure 2(b) shows that it is period-3 motion when $\xi \in(1.43,1.46)$ and generates saddle bifurcation (SNB) at $\xi=1.46$. The phase diagram of $\xi=1.44$ is shown as Figure 3(a). With changing the parameter $\xi$ in
$(0,1.42),(1.47,1.76),(3.42,3.49)$, and $(3.96,4.65)$, the fourwing memristive system is chaotic and the corresponding largest Lyapunov exponent is positive. Figure 3(b) is the phase diagram of $\xi=1.68$, and this system diverges in $\xi \epsilon$ $(4.66,4.8)$.

3.1.2. The Effect of Initial Condition Changing. The memristor-based chaotic system is extremely sensitive to initial values, and its dynamic characteristics directly transform with the variations of initial conditions. Fix the circuit parameters as $a=-0.5, b=2.4, \alpha=15, \beta=8, \xi=$ 1.68 , and $\gamma=15.15$. The initial values $\left(0.1,0.1,0, w_{1 o}\right)$ are selected as initial conditions, where $w_{1 o}$ is initial value of the state variable $w_{1}$. The Lyapunov exponent spectrum of system in (1) varying with initial condition $l_{1}=w_{1 o}$ is shown in Figure 4(a), and the corresponding bifurcation diagram for $y(t)$ is illustrated as Figure 4(b) with $l_{1} \in[-5,5]$.

The multistability refers to a variety of stable states generating with changing initial values under the same system 


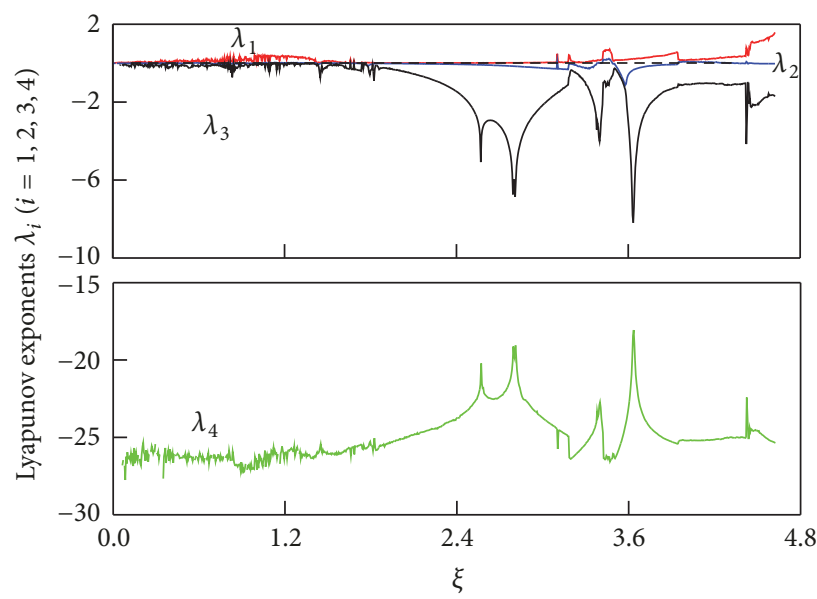

(a)

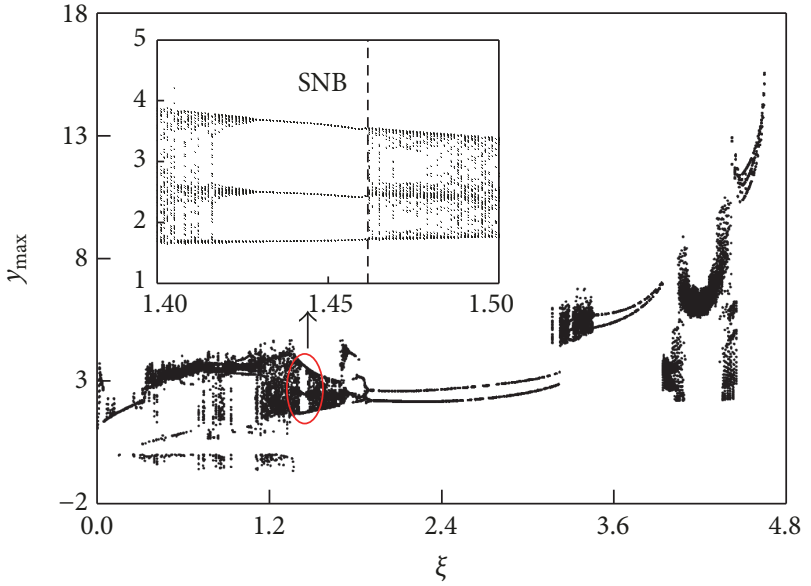

(b)

FIGURE 2: Lyapunov exponents and bifurcation diagram with parameter $\xi$ changing (a) $l_{1}=0$, Lyapunov exponents, and (b) $l_{1}=0$, bifurcation diagram.

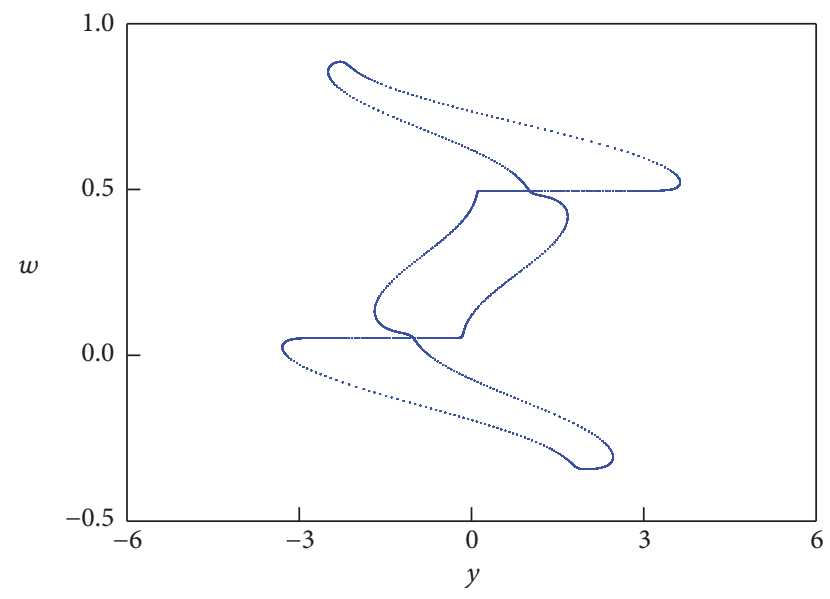

(a)

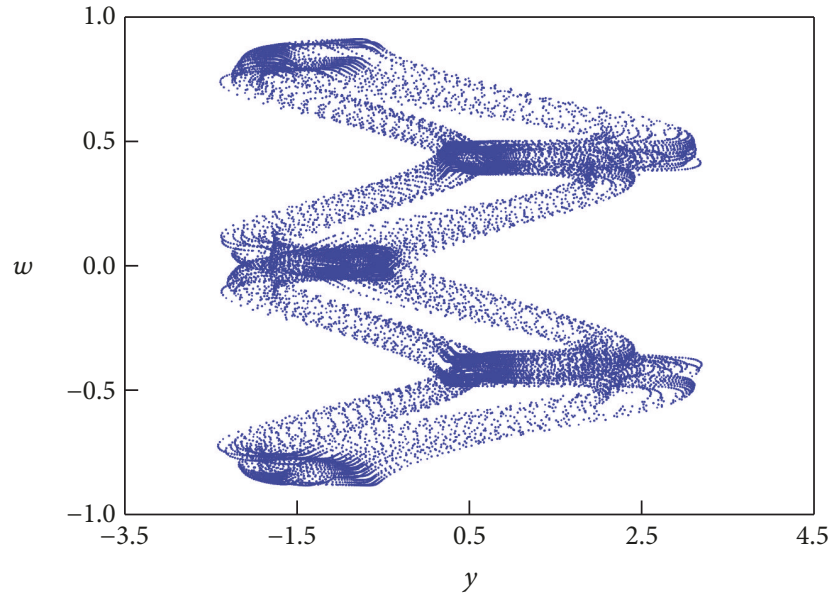

(b)

FIgURE 3: Phase diagrams of different system parameters $\xi$ : (a) $\xi=1.44$ and (b) $\xi=1.68$.

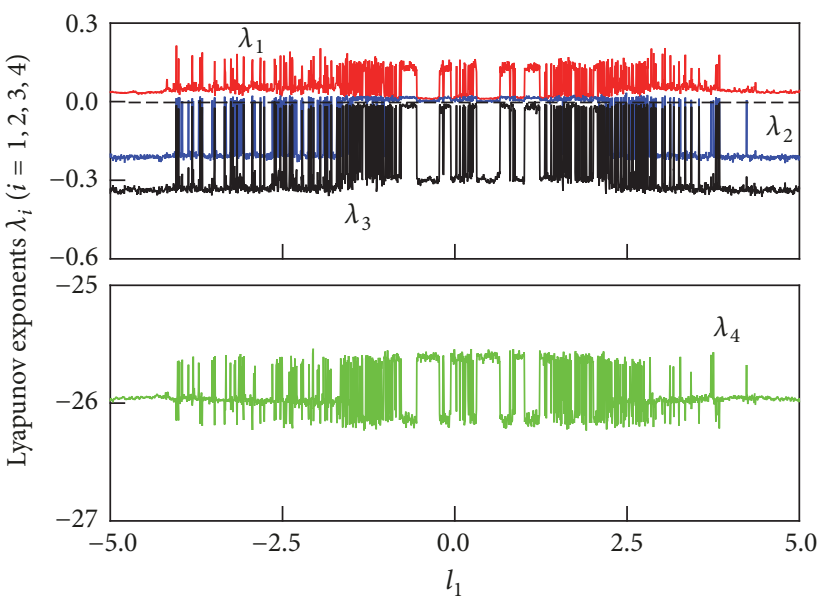

(a)

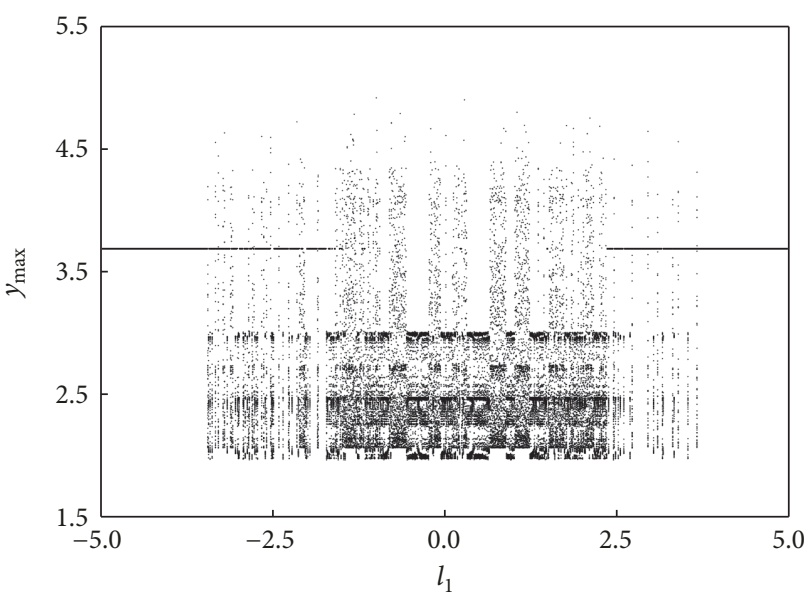

(b)

FiguRE 4: Lyapunov exponents and bifurcation diagram with parameter $l_{1}$ changing (a) $\xi=1.68$, Lyapunov exponents, and (b) $\xi=1.68$, bifurcation diagram. 


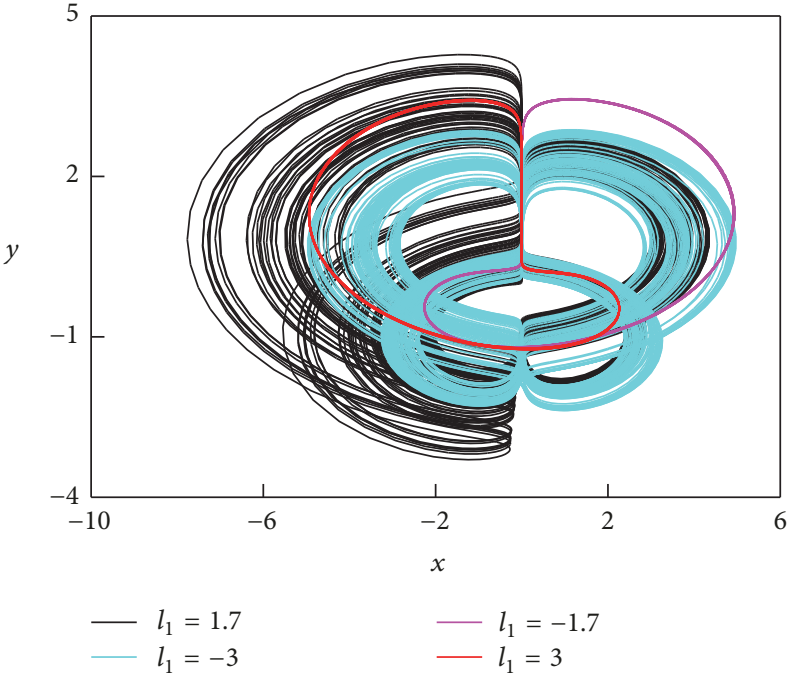

(a)

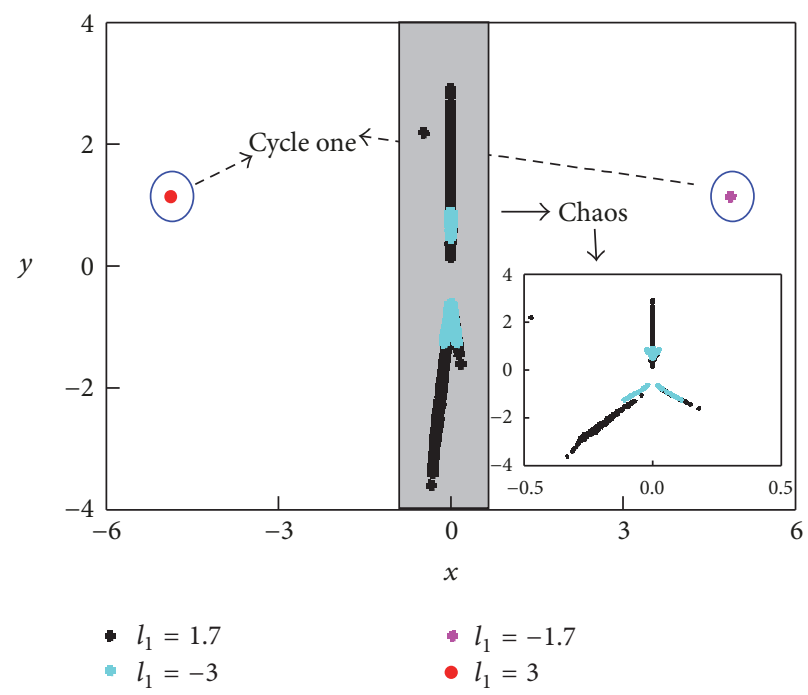

(b)

FIgURE 5: Coexistence of initial value $l_{1}$ changing (a) coexistence phase diagram and (b) coexistence of Poincaré section.

parameters. Figure 4 reveals that the chaotic system in (1) has a strong sensitivity to the transformation of $l_{1}$. The dynamic behaviors of system in (1) appear as the switch of periodic and chaotic motions with the variable $l_{1}$ varying. To observe the multistability clearly, select partial values for analyses randomly. Figure 5(a) shows the coexistence of four phase diagrams with different initial conditions. The black curves correspond to asymmetric double scrolls for $l_{1}=1.7$, and the light blue curves are associated with a butterfly attractor for $l_{1}=-3$. In addition, the pink and red curves are relative to period- 1 with $l_{1}=-1.7$ and $l_{1}=3$, respectively. Both periodic states are symmetrical about the $x$-axis. The corresponding coexistence of Poincaré sections is depicted in Figure 5(b). The gray domain means the chaotic region as $l_{1}=1.7$ (black points) and $l_{1}=-3$ (light blue points). The local gray area $(x \in[-0.5,0.5])$ is enlarged to observe the discrete mapping on Poincaré section clearly. The several successive curves can be seen from the partial magnification, proving that system in (1) is indeed in chaotic motion. Each of the two-blue ellipses in Figure 5(b) has a discrete point, corresponding to period1 as $l_{1}=3$ (red) and $l_{1}=-1.7$ (pink). Two discrete points are also symmetrical about the $x$-axis. Taking the length of the article into consideration, only four coexistence states are listed here.

3.1.3. Attraction Basins. The attraction basin is the distribution of different states and it has been a hot topic in the research on multiple attractors. In $[9,30]$, the attraction basins were carried out with respect to initial conditions. Similarly, the domains of initial conditions are analyzed in which each attraction can be found. The system parameters in (1) are set as $a=-0.5, b=2.4, \alpha=15, \beta=8, \xi=1.68$, and $\gamma=15.15$, and the initial values are assigned as $x_{1}(0)=0.1$, $y_{1}(0)=0.1, z_{1}(0)=p, w_{1}(0)=l_{1}$, where $p$ and $l_{1}$ are the variables. The attraction basins with respect to $p$ and $l_{1}$ are shown in Figure 6(a) which presented with the periods (purple) and chaotic attraction (red).

In addition, the basins of attractions are investigated with respect to initial condition $l_{1}$ and system parameter $\xi$. The parameters are set as $a=-0.5, b=2.4, \alpha=15, \beta=8, \gamma=$ 15.15 and initial values are given as $x_{1}(0)=0.1, y_{1}(0)=0.1$, $z_{1}(0)=0, w_{1}(0)=l_{1}$. The initial condition $l_{1}$ and system parameter $\xi$ are taken together to analyze the attraction basins shown in Figure 6(b), which is also associated with the periods (purple) and chaotic attraction (red). The boundaries are marked by yellow in Figure 6 and they conform with the analysis above; for example, the periodic motion (purple) as $l_{1}=3$ and $p=0$ in Figure 6 (a) corresponds to the red curves in Figure 5(a) and the chaotic motion (red) as $\xi=1.68$ and $l_{1}=0$ observed in Figure 6(a) matches the phase diagram shown in Figure 3(b), which means that the phenomenon of multistability indeed coexists in the proposed four-wing memristive chaotic system.

3.2. Circuit Implementation. The dynamic analysis of the four-wing memristive chaotic system shows its rich motion states. The circuit construction of system in (1) has further verified its complex dynamic behaviors by numerical simulations with the software MULTISIM.11. The modular design of the equivalent circuit is made up of basic components, such as resistors, capacitors, op amps, and multipliers, as shown in Figure 8. There are five channels: the first channel aims to achieve the function of memristor and the other four channels output $x, y, z$, and $w$, respectively. On the basis of Figure 8 and circuit principle, the circuit realization equation of four-wing memristive chaotic system in (1) is shown in (8):

$$
\begin{aligned}
& \frac{d x}{d t}=-\frac{1}{10 R_{6} C_{1}} z M-\frac{1}{10 R_{7} C_{1}}(-y z)-\frac{1}{R_{8} C_{1}} x, \\
& \frac{d y}{d t}=-\frac{1}{10 R_{11} C_{2}} x z-\frac{1}{R_{12} C_{2}}(-y),
\end{aligned}
$$




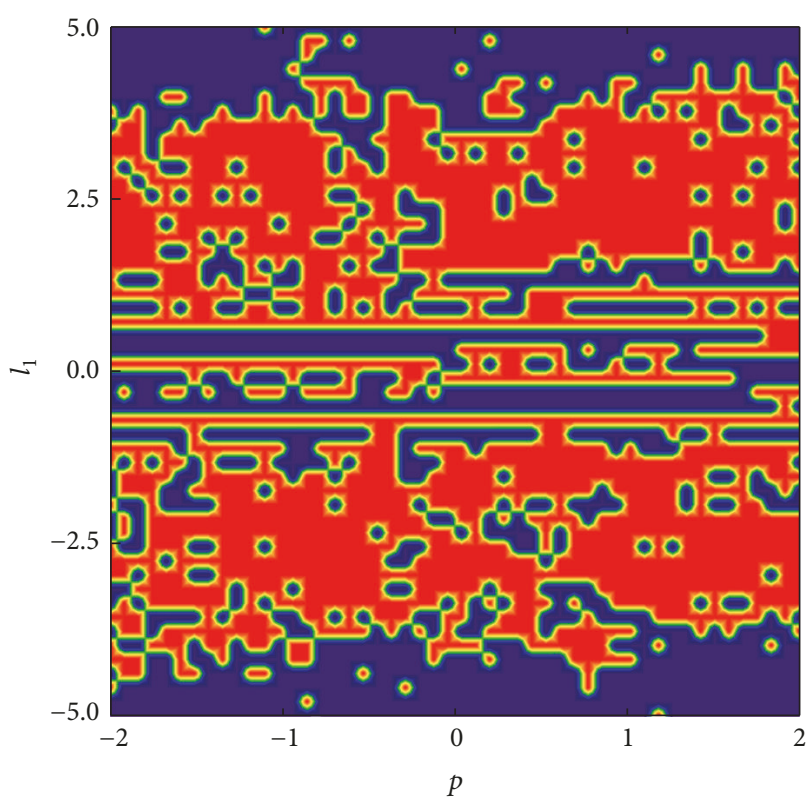

(a)

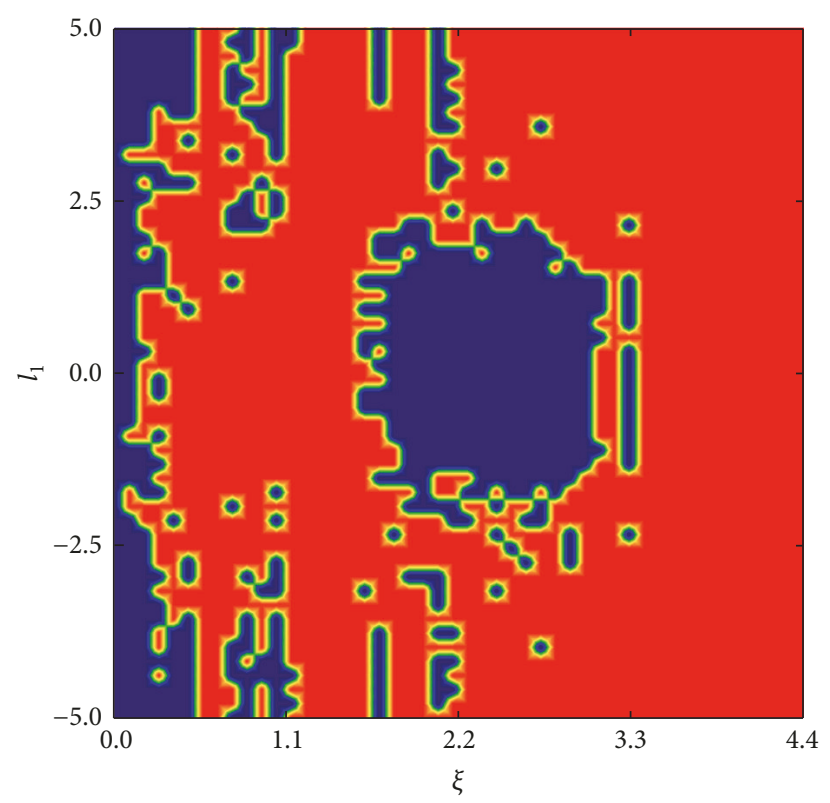

(b)

FIGURE 6: Attraction basins: (a) under initial conditions $\left(0.1,0.1, p, l_{1}\right)$ and (b) under initial condition $l_{1}$ and system parameter $\xi$.

$$
\begin{aligned}
& \frac{d z}{d t}=-\frac{1}{10 R_{15} C_{3}}(-x y)-\frac{1}{R_{16} C_{3}} z \\
& \frac{d w}{d t}=-\frac{1}{R_{17} C_{4}}(-x) .
\end{aligned}
$$

Comparing (1) and (8), the parameters of the expression can be obtained:

$$
\begin{aligned}
\frac{1}{R_{8} C_{1}} & =\alpha, \\
\frac{1}{10 R_{11} C_{2}} & =\beta, \\
\frac{1}{R_{12} C_{2}} & =\xi, \\
\frac{1}{R_{16} C_{3}} & =\gamma, \\
\frac{1}{10 R_{7} C_{1}} & =36, \\
\frac{1}{10 R_{15} C_{3}} & =8, \\
\frac{1}{10 R_{6} C_{1}} & =\frac{1}{R_{17} C_{4}}=1 .
\end{aligned}
$$

Let $\alpha=15, \beta=8, \xi=1.68$, and $\gamma=15.15$; the values of four capacitors in Figure 8 are taken as $C_{1}=C_{2}=C_{3}=$ $C_{4}=1 \mathrm{nF}$. The resistance values in circuit are calculated as $R_{1}=1 \mathrm{k} \Omega, R_{2}=48 \mathrm{k} \Omega, R_{3}=24 \mathrm{k} \Omega, R_{6}=100 \mathrm{k} \Omega$, $R_{7}=2.78 \mathrm{k} \Omega, R_{8}=66.7 \mathrm{k} \Omega, R_{11}=12.5 \mathrm{k} \Omega, R_{12}=596 \mathrm{k} \Omega$,
$R_{15}=16.7 \mathrm{k} \Omega, R_{16}=67 \mathrm{k} \Omega, R_{17}=1 \mathrm{M} \Omega, R_{4}=10 \mathrm{k} \Omega$, $R_{5}=R_{9}=R_{10}=10 \mathrm{k} \Omega, R_{13}=R_{14}=10 \mathrm{k} \Omega$. The value of system parameter $\xi$ is determined by resistor $R_{12}$, and the different motions can be got by adjusting the value of $R_{12}$. The resistance $R_{12}$ can be accessed by a potentiometer in the actual circuit. Take $R_{12}=596 \mathrm{k} \Omega, C_{2}=1 \mathrm{nF}$ when $\xi=1.68$, then the circuit simulation result is illustrated in Figure $7(\mathrm{a})$. The coexistence phase diagrams are shown as Figure 7(b) with the variation of $l_{1}$. Only two cases $l_{1}=-3$ (light blue) and $l_{1}=$ -1.7 (pink) are listed here. It is possible to show that there is a multistability characteristic in system in (1) as well. The result in Figure $7(a)$ is in accordance with phase diagram plotted in Figure 1(b), and coexistence phase diagrams shown in Figure $7(\mathrm{~b})$ agree with the light blue points $\left(l_{1}=-3\right)$ and pink points $\left(l_{1}=-1.7\right)$ shown in Figure $5(\mathrm{a})$. Hence the results of circuit and numerical simulation basically correspond under the same conditions.

\section{Synchronization of the Memristive System and Its Application in Image Encryption}

In 1990, a chaotic synchronization control method was firstly proposed by Pecora and Carroll [31]. Then, lots of results of chaos synchronization have been taken by scholars [32, 33]. With the gradual development of memristor, some scholars have applied the synchronization control to memristive chaotic systems, which has aroused a new upsurge in people's research. The nonlinear controller is designed according to this new four-dimensional four-wing memristive chaotic system for completing the synchronization of multistability. Moreover, the reliability of secret keys has been improved and the security of encryption has been enhanced by applying it to the image encryption. 


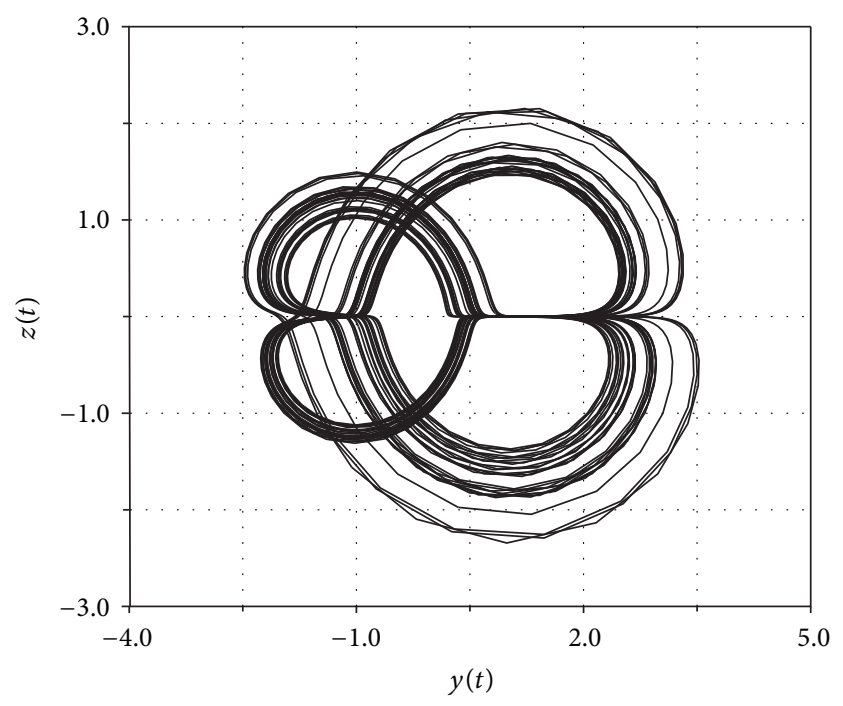

(a)

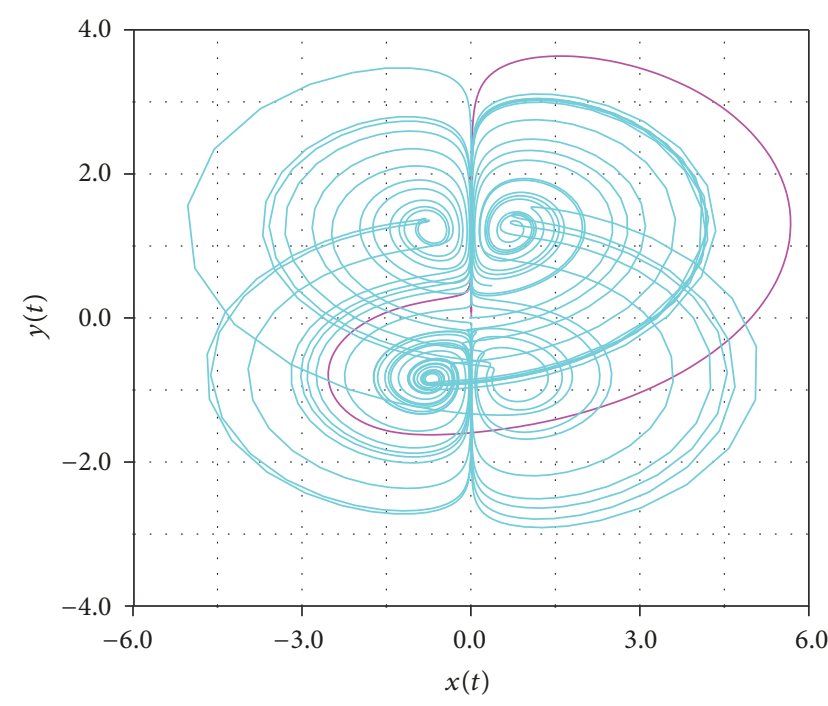

(b)

FIGURE 7: Circuit simulation diagrams: (a) $y$ - $z$ phase diagram and (b) $x-y$ coexistence phase diagrams.

4.1. Synchronization of Four-Wing Memristive Chaotic System. According to the dynamic analysis of this four-wing memristive chaotic system, multistability will appear with initial conditions varying. A reasonable controller is devised to achieve the synchronization for coexistence attractors. Equation (1) is set as master system, so slave system is given by

$$
\begin{aligned}
& \dot{x}_{2}=36 y_{2} z_{2}-\alpha x_{2}-z_{2} W\left(w_{2}\right)+u_{1}, \\
& \dot{y}_{2}=\xi y_{2}-\beta x_{2} z_{2}+u_{2}, \\
& \dot{z}_{2}=8 x_{2} y_{2}-\gamma z_{2}+u_{3}, \\
& \dot{w}_{2}=x_{2}+u_{4},
\end{aligned}
$$

where $W\left(w_{2}\right)=a+b w_{2}^{2}, a=-0.5, b=2.4, \alpha=15, \beta=8$, $\xi=1.68$, and $\gamma=15.15 . u_{1}, u_{2}, u_{3}$, and $u_{4}$ are synchronization controllers. The synchronization error is described as

$$
\begin{aligned}
& e_{1}=x_{2}-x_{1}, \\
& e_{2}=y_{2}-y_{1}, \\
& e_{3}=z_{2}-z_{1}, \\
& e_{4}=w_{2}-w_{1} .
\end{aligned}
$$

The equation of error system in (11) is

$$
\begin{aligned}
\dot{e}_{1}= & 36\left(y_{2} z_{2}-y_{1} z_{1}\right)-15 e_{1} \\
& -\left[z_{2}\left(-0.5+2.4 w_{2}^{2}\right)-z_{1}\left(-0.5+2.4 w_{1}^{2}\right)\right] \\
& +u_{1}, \\
\dot{e}_{2}= & 1.68 e_{2}-8\left(x_{2} z_{2}-x_{1} z_{1}\right)+u_{2}, \\
\dot{e}_{3}= & 8\left(x_{2} y_{2}-x_{1} y_{1}\right)-15.15 e_{3}+u_{3}, \\
\dot{e}_{4}= & e_{1}+u_{4} .
\end{aligned}
$$

Theorem 1. Select the following nonlinear feedback synchronization controller functions:

$$
\begin{aligned}
u_{1}= & -k_{1}{ }^{\left|e_{1}\right|} e_{1}+0.5 e_{3}-36\left(y_{2} z_{2}-y_{1} z_{1}\right) \\
& -2.4\left(z_{2} w_{2}{ }^{2}-z_{1} w_{1}^{2}\right), \\
u_{2}= & -k_{2}^{\left|e_{2}\right|} e_{2}-2 e_{2}+8\left(x_{2} z_{2}-x_{1} z_{1}\right), \\
u_{3}= & -k_{3}{ }^{\left|e_{3}\right|} e_{3}-8\left(x_{2} y_{2}-x_{1} y_{1}\right), \\
u_{4}= & -k_{4}{ }^{\left|e_{4}\right|} e_{4}-e_{1} .
\end{aligned}
$$

Master system in (1) is asymptotically synchronized with slave system in (10) when the feedback control gain $k_{i}(i=$ $1,2,3,4) \geq 0$.

Proof. Synchronization controller in (13) is designed with the specific form of error system in (12). The expressions with $-k_{i}^{\left|e_{i}\right|} e_{i}(i=1,2,3,4)$ [34] are added to increase the speed of synchronization and reduce the synchronization error in transition process. The error system can be simplified from (12) and (13) as

$$
\begin{aligned}
& \dot{e}_{1}=-k_{1}{ }^{\left|e_{1}\right|} e_{1}-15 e_{1}, \\
& \dot{e}_{2}=-k_{2}{ }^{\left|e_{2}\right|} e_{2}-0.32 e_{2}, \\
& \dot{e}_{3}=-k_{3}{ }^{\left|e_{3}\right|} e_{3}-15.15 e_{3}, \\
& \dot{e}_{4}=-k_{4}{ }^{\left|e_{4}\right|} e_{4} .
\end{aligned}
$$

Construct the Lyapunov function as $V=(1 / 2) e^{T} e$, and the derivative of $V$ is calculated as

$$
\begin{aligned}
\dot{V}= & \dot{e}^{T} e=e_{1} \dot{e}_{1}+e_{2} \dot{e}_{2}+e_{3} \dot{e}_{3}+e_{4} \dot{e}_{4} \\
= & -k_{1}{ }^{\left|e_{1}\right|} e_{1}{ }^{2}-15 e_{1}{ }^{2}-k_{2}{ }^{\left|e_{2}\right|} e_{2}{ }^{2}-0.32 e_{2}{ }^{2}-k_{3}{ }^{\left|e_{3}\right|} e_{3}{ }^{2} \\
& -15.15 e_{3}{ }^{2}-k_{4}{ }^{\left|e_{4}\right|} e_{4}{ }^{2}
\end{aligned}
$$




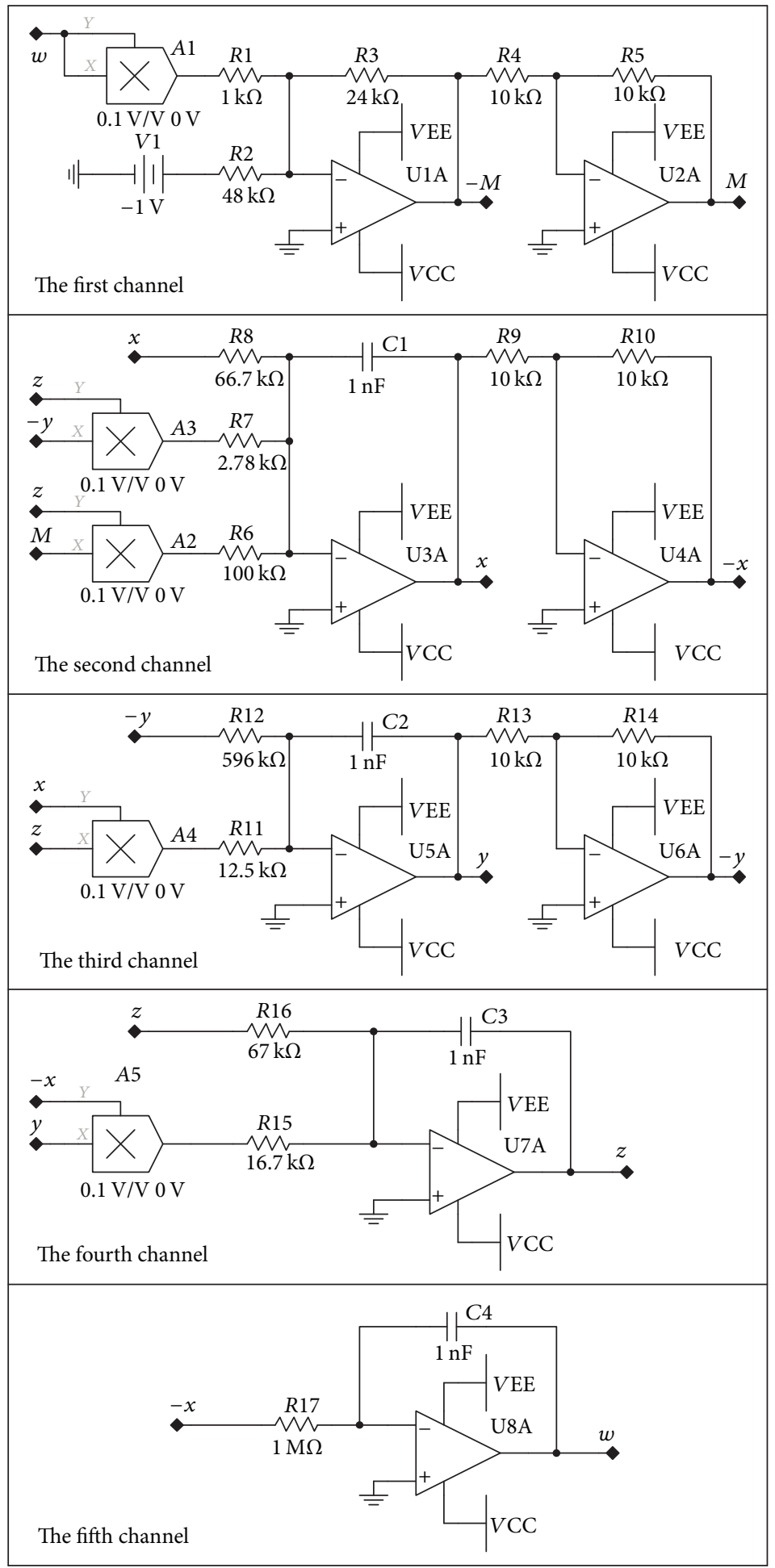

FIGURE 8: Circuit schematic.

$$
\begin{aligned}
= & -\left(k_{1}^{\left|e_{1}\right|} e_{1}^{2}+k_{2}{ }^{\left|e_{2}\right|} e_{2}^{2}+k_{3}{ }^{\left|e_{3}\right|} e_{3}{ }^{2}+k_{4}{ }^{\left|e_{4}\right|} e_{4}{ }^{2}\right) \\
& -\left(15 e_{1}^{2}+0.32 e_{2}^{2}+15.15 e_{3}^{2}\right) .
\end{aligned}
$$

It is obvious that $\dot{V}$ is negative owing to $k_{i}(i=1,2,3,4) \geq$ 0 , and the error system in (12) is large-scale asymptotic stable. Master system (1) and slave system (10) can be synchronized under the nonlinear synchronization controller in (13). 


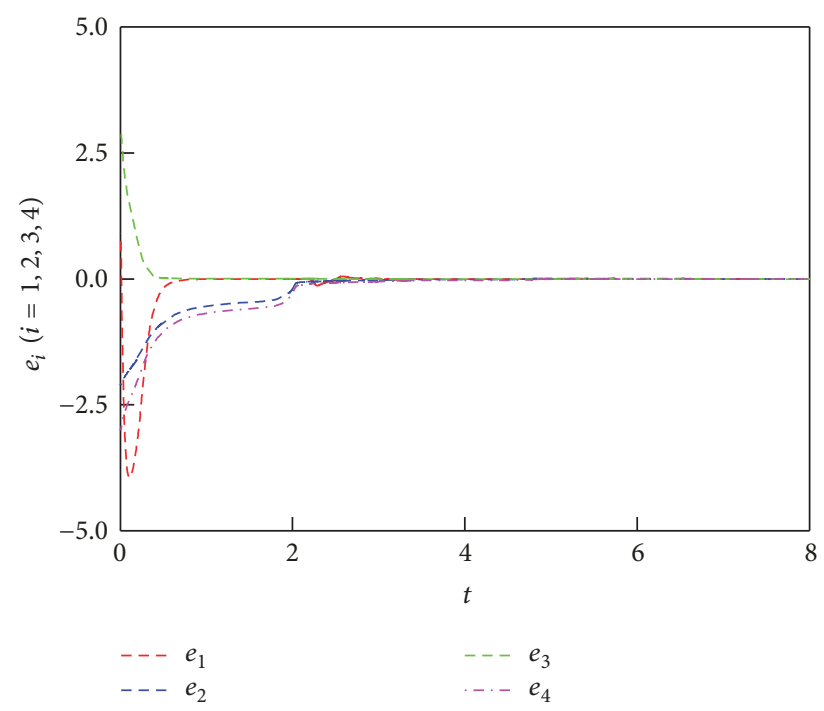

FIGURE 9: Synchronization errors.

According to the proof afore-discussed, the feedback gain is chosen as $k_{i}(i=1,2,3,4)=2$ randomly. $\left(x_{1}, y_{1}, z_{1}, w_{1}, x_{2}, y_{2}, z_{2}, w_{2}\right)=(0.1,0.1,0,0,1,-2,3,-3)$ is set to be the initial values of system (1) and system (10). The response curves of system errors are shown in Figure 9, and the synchronization errors converge to zero at about $4 \mathrm{~s}$.

To realize the synchronization of multiple dynamics in the four-wing memristive chaotic system, initial conditions of master system in (1) are set to $\left(0.1,0.1,0, w_{10}\right)$ and those of slave system in $(10)$ are taken as $\left(0.1,0.1,0, w_{2 o}\right)$. It is clear that the first three terms of two initial conditions remain unified, and the fourth terms $w_{1 o}$ and $w_{2 o}$ are set as variables. According to the aforementioned dynamics analyses above, the system in (1) will produce a rich multistable phenomenon along with initial condition $l_{1}=w_{1 o}$ varying. The motion of master system is a chaotic attractor as shown in Figure 1 as $l_{1}=0$, and it is in period- 1 motion with the red points in Figure 5(b) as $l_{1}=3$. Similarly, the same coexistence attractors can be observed with the variable $l_{2}=w_{2 o}$ without regarding the influence of nonlinear synchronization controllers. The following two cases will be used to demonstrate the synchronization of coexistence states in the four-wing memristive chaotic system.

Case 1: setting two variables as $l_{1}=0$ and $l_{2}=3$, slave system in (10) can be synchronized with master system in (1) as a chaotic state. The timing diagrams for variables $l_{1}$ and $l_{2}$ are depicted in Figure 10(a). Similarly, case 2: for the variables above obtained as $l_{1}=3$ and $l_{2}=0$, the slave and master systems are synchronized as a periodic state. The state evolutions in this case are shown in Figure 10(b). Figure 10 indicates that these two cases are fully synchronized at about $4 \mathrm{~s}$; that is, the multi-steady-state synchronization of the four-wing memristive chaotic system can be realized. Both different synchronization effects lay foundation for the application of memristive chaotic synchronization in image encryption.
4.2. Image Encryption. Chaotic system can provide sequences for information encryption, and most of the current researches on secure communication are for chaotic system without memristor $[35,36]$. As we all know, the memristive chaotic circuit has a complex topology which can extend the dimension of chaotic systems, and it is extremely sensitive to the variation of initial condition that the better sequence can be extracted. So far, there were few studies on the application of synchronization of memristive chaotic system in image encryption. The initial conditions of master system in (1) and slave system in (8) are used as keys to improve the complexity of secret keys and anticrack ability. Figure 11 shows the process of encryption and decryption. A chaotic synchronization control signal is generated by the four-wing chaotic system to encrypt the original image, and the discrete sequences are also obtained from it. The encrypted image is got by the encryption transformation of discrete sequences and original image. The encrypted image will be transformed to the decryption terminal through four channels, including all data of the encrypted image and the data in each channel with unequal quantity to enhance the difficulty of deciphering. The decryption terminal receives encrypted signals and generates the decrypted image according to the key and encryption transformation, completing the synchronization of four-wing memristive chaotic system applied in image encryption.

The typical image "cameraman" [37] is selected for image encryption analysis, and the original image and its histogram are presented in Figure 12. According to the above analysis of the synchronization method, for the first case, initial conditions of master system (1) and slave system (10) are taken as $(0.1,0.1,0,0)$ and $(0.1,0.1,0,3)$, respectively. The synchronization result of master and slave system is chaos, and the encrypted image and its histogram are shown in Figure 13. The encrypted image exhibits a fuzzy state, and there is no feature of the original image. The histogram exhibited in Figure 13(b) no longer has the statistical characteristics of original image, so that the effect of encryption is good. The same method is utilized for the second case, and the initial values of system in (1) and system in (10) are, respectively, sited as $(0.1,0.1,0,3)$ and $(0.1,0.1,0,0)$. Thus, the result of synchronization is periodic motion in this case, and the encrypted image and its histogram are plotted in Figure 14. Contrasting Figure 14 with Figures 12 and 13, the encrypted image depicted in Figure 14(a) retains some features of original image exhibited in Figure 12(a). Similarly, the histogram shown in Figure 14(b) still has some statistical features of the original image, and the encryption is flawed. Therefore, the synchronization control of different motions will have different effects on image encryption, playing an important role in the study of secure communication.

On the basis of different effects on image encryption, it is better to apply case 1 to image encryption so that the image encryption with case 1 will be considered to analyze the noise attack. It is a common phenomenon to add noise in the process of information transmission. The Gaussian noise with the percentage of $1 \%$ is added to the encrypted image of case 1 , and the decrypted image is depicted in Figure 15(a). Similarly, the salt-and-pepper noise with the percentage of $2 \%$ is put in 


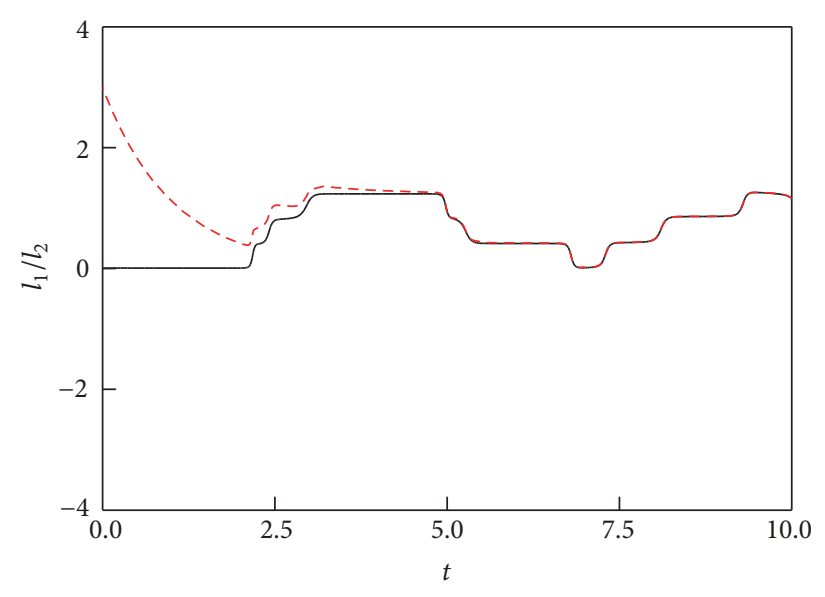

$-l_{1}$

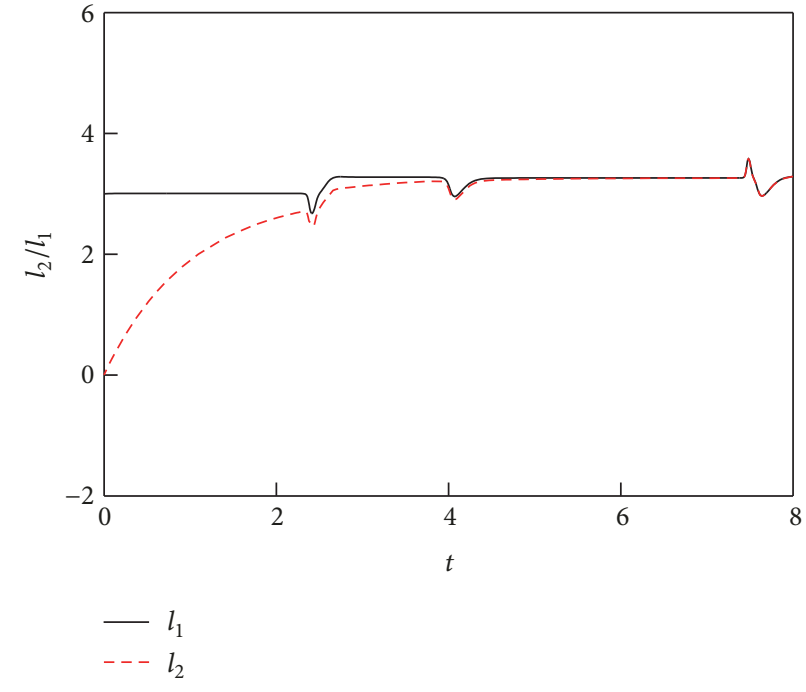

(b)

Figure 10: Timing diagrams: (a) case $1, l_{1}=0 \& l_{2}=3$, and (b) case $2, l_{1}=3 \& l_{2}=0$.

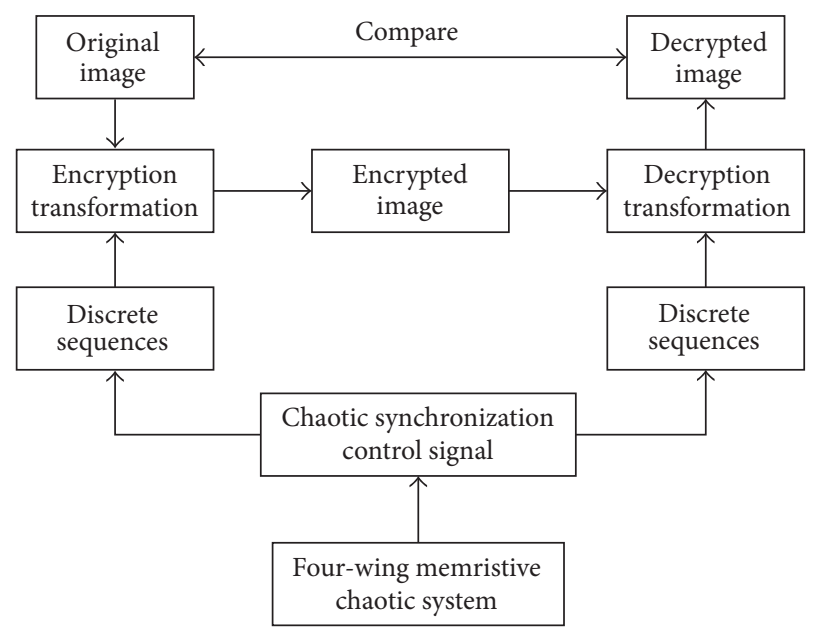

FIGURE 11: Encryption and decryption flow chart.

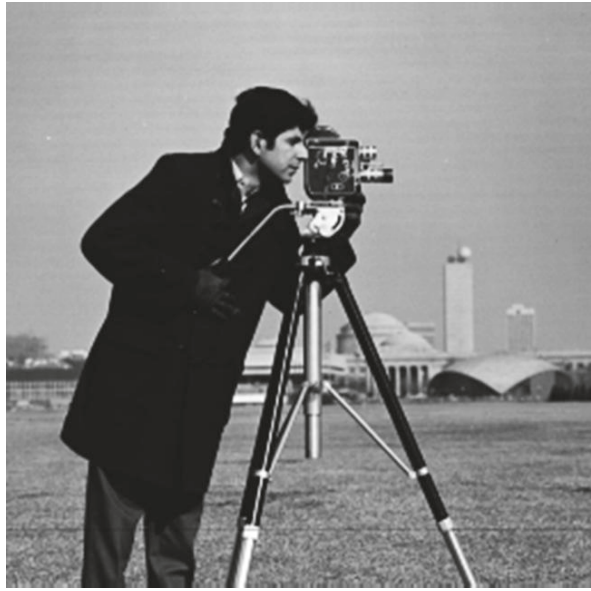

(a)

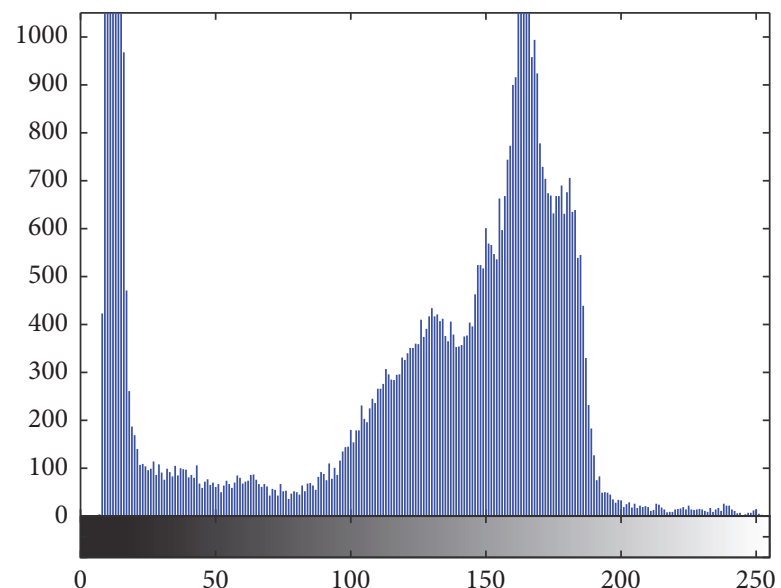

(b)

FIgURE 12: Typical image "cameraman": (a) original image and (b) corresponding histogram. 


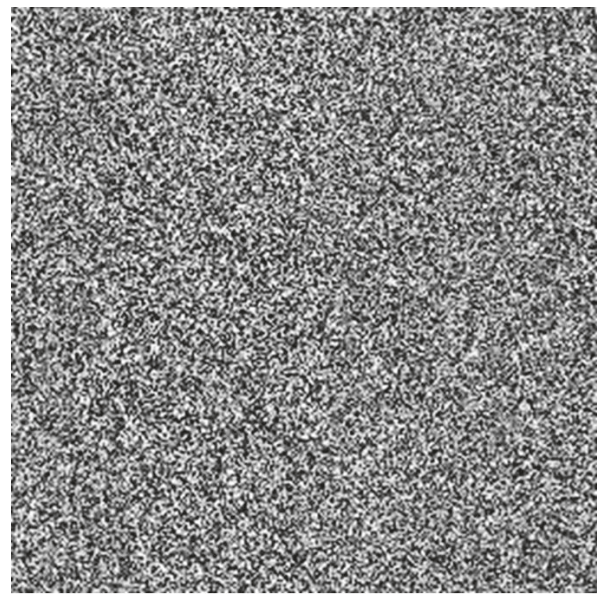

(a)

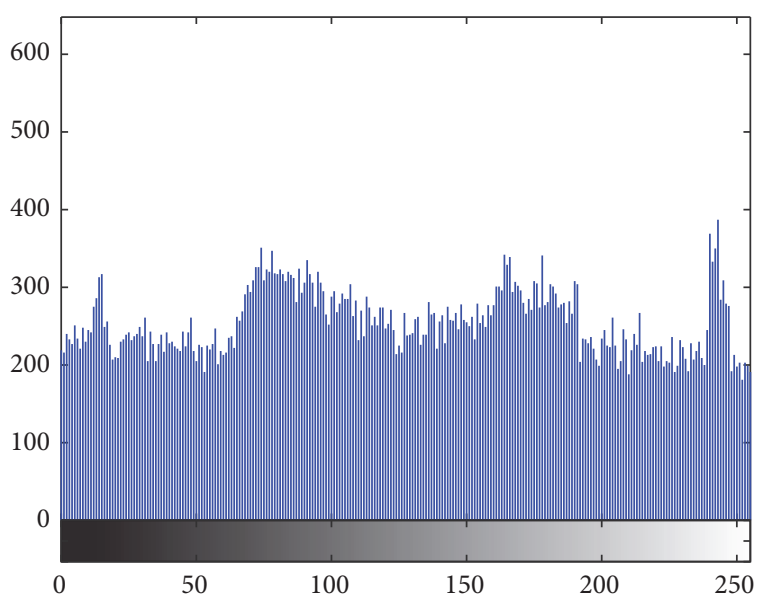

(b)

FIGURE 13: Case 1: (a) encrypted image and (b) encrypted histogram.

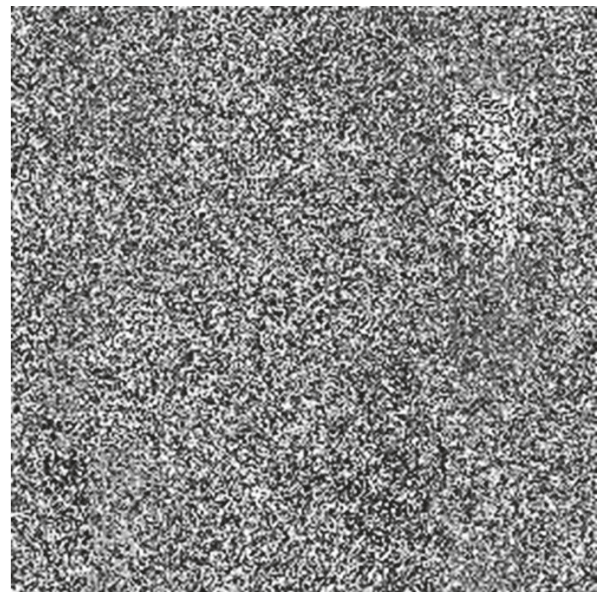

(a)

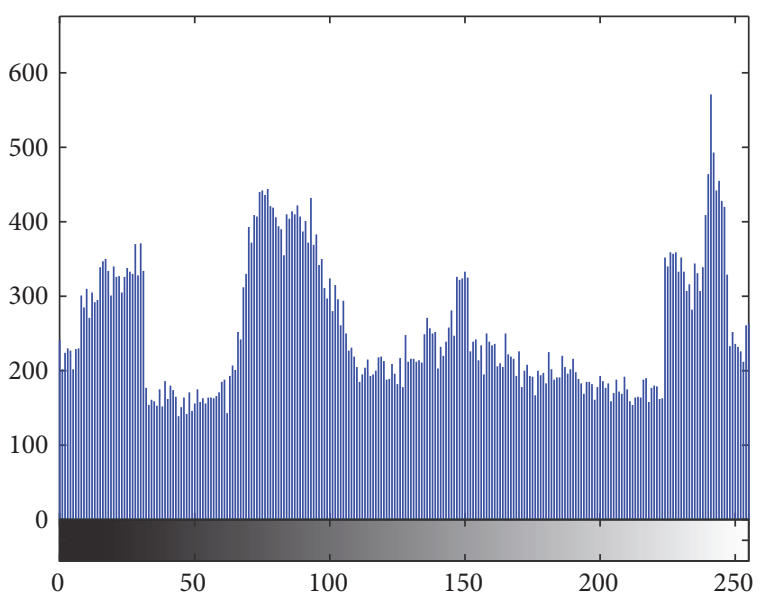

(b)

FIGURE 14: Case 2: (a) encrypted image and (b) encrypted histogram.

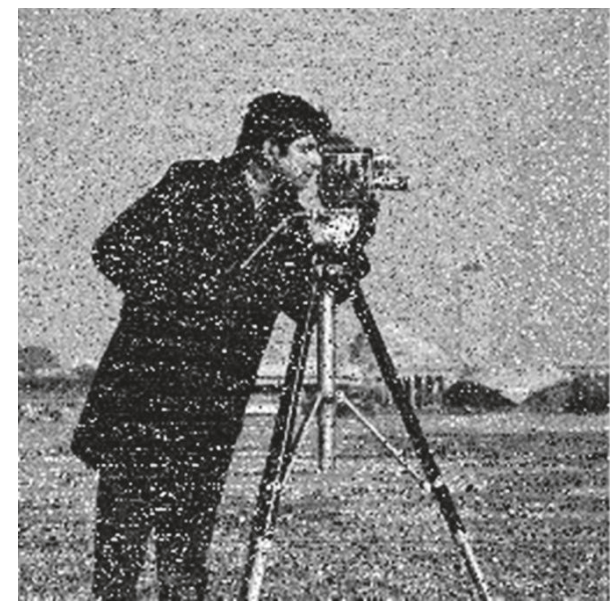

(a)

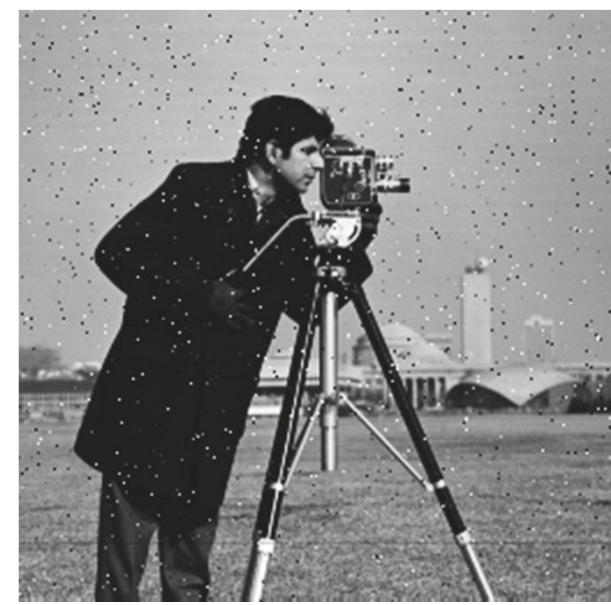

(b)

FIGURE 15: Decrypted images: (a) attacked by Gaussian noise and (b) attacked by salt-and-pepper noise. 
the encrypted image in Figure 13, and the decrypted image is exhibited in Figure 15(b). Although noise interference can be observed in decrypted images, the main information of original image presented in Figure 12(a) remains. The good decryption effects prove that the encryption algorithm can effectively resist different noise attacks.

\section{Conclusion}

In summary, a modified Lorenz system is obtained on the basis of classical continuous Lorenz chaotic system, and a new four-dimensional four-wing memristive chaotic system is proposed by the addition of a smooth active flux-controlled memristor. The rich dynamical behaviors are exhibited through Lyapunov exponents, bifurcation diagrams, coexistence Poincaré sections, coexistence phase diagrams, and attraction basins. The multistability can be observed with respect to the sensitivity of initial conditions diametrically. Moreover, a new nonlinear synchronization controller with exponential term is designed to realize the synchronization control of multiple attractors and applied to image encryption. The chaotic sequence based on the synchronization scheme is extracted to increase the complexity of keys. At the same time, it is analyzed that the keys generated in different states have an important impact on encryption, and the choice of appropriate key is valuable to improve security.

\section{Conflicts of Interest}

The authors declare that there are no conflicts of interest regarding the publication of this paper.

\section{Acknowledgments}

The work is supported by the National Natural Science Foundation of China (no. 51475246), the Natural Science Foundation of Jiangsu Province under Grant no. Bk20131402, and the Postgraduate Research \& Practice Innovation Program of Jiangsu Province of China under Grant no. KYCX17_1082.

\section{References}

[1] L. O. Chua, "Memristor-the missing circuit element," IEEE Transactions on Circuit Theory, vol. 18, no. 5, pp. 507-519, 1971.

[2] D. B. Strukov, G. S. Snider, D. R. Stewart, and R. S. Williams, "The missing memristor found," Nature, vol. 453, pp. 80-83, 2008.

[3] J. J. Yang, D. B. Strukov, and D. R. Stewart, "Memristive devices for computing," Nature Nanotechnology, vol. 8, no. 1, pp. 13-24, 2013.

[4] C. Yakopcic, V. Bontupalli, R. Hasan, D. Mountain, and T. M. Taha, "Self-biasing memristor crossbar used for string matching and ternary content-addressable memory implementation," IEEE Electronics Letters, vol. 53, no. 7, pp. 463-465, 2017.

[5] Y. Chang, F. Zhou, B. W. Fowler et al., "Memcomputing (Memristor + Computing) in intrinsic SiOx-based resistive switching memory: arithmetic operations for logic applications," IEEE Transactions on Electron Devices, vol. 64, no. 7, pp. 2977-2983, 2017.
[6] L. Wang, Q. Song, Y. Liu, Z. Zhao, and F. E. Alsaadi, "Finitetime stability analysis of fractional-order complex-valued memristor-based neural networks with both leakage and timevarying delays," Neurocomputing, vol. 245, pp. 86-101, 2017.

[7] J. Feng, Q. Ma, and S. Qin, "Exponential stability of periodic solution for impulsive memristor-based Cohen-Grossberg neural networks with mixed delays," International Journal of Pattern Recognition and Artificial Intelligence, vol. 31, no. 7, 1750022, 17 pages, 2017.

[8] R. Rocha, J. Ruthiramoorthy, and T. Kathamuthu, "Memristive oscillator based on Chua's circuit: stability analysis and hidden dynamics," Nonlinear Dynamics, vol. 88, no. 4, pp. 2577-2587, 2017.

[9] J. Kengne, A. N. Negou, and D. Tchiotsop, "Antimonotonicity, chaos and multiple attractors in a novel autonomous memristor-based jerk circuit," Nonlinear Dynamics, vol. 88, no. 4, pp. 2589-2608, 2017.

[10] H. Abunahla, D. Shehada, C. Y. Yeun, B. Mohammad, and M. A. Jaoude, "Novel secret key generation techniques using memristor devices," AIP Advances, vol. 6, no. 2, Article ID 025107, 2016.

[11] S. Kannan, N. Karimi, O. Sinanoglu, and R. Karri, "Security Vulnerabilities of Emerging Nonvolatile Main Memories and Countermeasures," IEEE Transactions on Computer-Aided Design of Integrated Circuits and Systems, vol. 34, no. 1, pp. 2-15, 2015.

[12] Z. Wang, F. Min, and E. Wang, "A new hyperchaotic circuit with two memristors and its application in image encryption," AIP Advances, vol. 6, no. 9, Article ID 095316, 2016.

[13] Q. Xu, Y. Lin, B. Bao, and M. Chen, "Multiple attractors in a nonideal active voltage-controlled memristor based Chua's circuit," Chaos, Solitons \& Fractals, vol. 83, pp. 186-200, 2016.

[14] H. Xi, Y. Li, and X. Huang, "Generation and nonlinear dynamical analyses of fractional-order memristor-based Lorenz systems," Entropy, vol. 16, no. 12, pp. 6240-6253, 2014.

[15] B. Zhang and F. Q. Deng, "Double-compound synchronization of six memristor-based Lorenz systems," Nonlinear Dynamics, vol. 77, no. 4, pp. 1519-1530, 2014.

[16] S. Wang, X. Wang, and Y. Zhou, "A memristor-based complex lorenz system and its modified projective synchronization," Entropy, vol. 17, no. 11, pp. 7628-7644, 2015.

[17] S. Kassim, H. Hamiche, S. Djennoune, and M. Bettayeb, "A novel secure image transmission scheme based on synchronization of fractional-order discrete-time hyperchaotic systems," Nonlinear Dynamics, vol. 88, no. 4, pp. 2473-2489, 2017.

[18] S. Wang, J. Zhao, X. Li, and L. Zhang, "Image blocking encryption algorithm based on laser chaos synchronization," Journal of Electrical and Computer Engineering, vol. 2016, Article ID 4138654, 14 pages, 2016.

[19] Z. Lin and H. Wang, "Image encryption based on chaos with PWL memristor in Chua's circuit," in Proceedings of the 2009 International Conference on Communications, Circuits and Systems (ICCCAS), pp. 964-968, Milpitas, Ca, USA, July 2009.

[20] C. Yang, Q. Hu, Y. Yu, R. Zhang, Y. Yao, and J. Cai, "MemristorBased Chaotic Circuit for Text/Image Encryption and Decryption," in Proceedings of the 8th International Symposium on Computational Intelligence and Design, ISCID 2015, pp. 447450, China, December 2015.

[21] J. Ma, Z. Chen, Z. Wang, and Q. Zhang, "A four-wing hyperchaotic attractor generated from a $4 \mathrm{D}$ memristive system with a line equilibrium," Nonlinear Dynamics, vol. 81, no. 3, pp. 12751288, 2015. 
[22] L. Zhou, C. Wang, and L. Zhou, "Generating Four-Wing Hyperchaotic Attractor and Two-Wing, Three-Wing, and Four-Wing Chaotic Attractors in 4D Memristive System," International Journal of Bifurcation and Chaos, vol. 27, no. 2, Article ID 1750027, 2017.

[23] N. H. Alombah, H. Fotsin, and K. Romanic, "Coexistence of multiple attractors, metastable chaos and bursting oscillations in a multiscroll memristive chaotic circuit," International Journal of Bifurcation and Chaos, vol. 27, no. 5, 1750067, 20 pages, 2017.

[24] Z. T. Njitacke, J. Kengne, H. B. Fotsin, A. N. Negou, and D. Tchiotsop, "Coexistence of multiple attractors and crisis route to chaos in a novel memristive diode bidge-based Jerk circuit," Chaos, Solitons \& Fractals, vol. 91, pp. 180-197, 2016.

[25] G. Peng and F. Min, "Multistability analysis, circuit implementations and application in image encryption of a novel memristive chaotic circuit," Nonlinear Dynamics, vol. 90, no. 3, pp. 16071625, 2017.

[26] E. N. Lorenz, "Deterministic nonperiodic flow," Journal of the Atmospheric Sciences, vol. 20, no. 2, pp. 130-141, 1963.

[27] S. M. Yv, Chaotic Systems and Chaotic Circuit: Principle, Design and Its Application in Communications, Xidian University Publishing House, Xi’an, China, 2011.

[28] G. A. Leonov, N. V. Kuznetsov, and T. N. Mokaev, "Hidden attractor and homoclinic orbit in Lorenz-like system describing convective fluid motion in rotating cavity," Communications in Nonlinear Science and Numerical Simulation, vol. 28, no. 1-3, pp. 166-174, 2015.

[29] H. Bao, B. C. Bao, Y. Lin et al., "Hidden attractor and its dynamical characteristic in memristive self-oscillating system," Acta Physica Sinica, vol. 65, no. 18, article 180501, 2016.

[30] B. Bao, T. Jiang, G. Wang, P. Jin, H. Bao, and M. Chen, “Twomemristor-based Chua's hyperchaotic circuit with plane equilibrium and its extreme multistability," Nonlinear Dynamics, pp. $1-15,2017$.

[31] L. M. Pecora and T. L. Carroll, "Synchronization in chaotic systems," Physical Review Letters, vol. 64, no. 8, pp. 821-824, 1990.

[32] F. Min and A. C. Luo, "Complex dynamics of projective synchronization of Chua circuits with different scrolls," International Journal of Bifurcation and Chaos, vol. 25, no. 5, 1530016, 28 pages, 2015.

[33] B. S. Reddy and A. Ghosal, "Chaotic Motion in a Flexible Rotating Beam and Synchronization," Journal of Computational and Nonlinear Dynamics, vol. 12, no. 4, p. 044505, 2017.

[34] L. Shan, J. Li, F. H. Min, and Z. Q. Wang, "Synchronization control of new piecewise fractional-order chaotic systems," Systems Engineering and Electronics, vol. 32, no. 10, pp. 21982202, 2010.

[35] C. L. Wang, Z. B. Chen, and G. E. Yong, "Infrared image encryption scheme using Lorenz chaotic system," Journal of Computer Applications, vol. 35, no. 8, pp. 2205-2209, 2015.

[36] J. Zhao, S. Wang, L. Zhang, and X. Wang, "Image encryption algorithm based on a novel improper fractional-order attractor and a wavelet function map," Journal of Electrical and Computer Engineering, vol. 2017, Article ID 8672716, 10 pages, 2017.

[37] I. F. Elashry, O. S. F. Allah, A. M. Abbas, S. El-Rabaie, and F. E. A. El-Samie, "Homomorphic image encryption," Journal of Electronic Imaging, vol. 18, no. 3, Article ID 033002, 2009. 


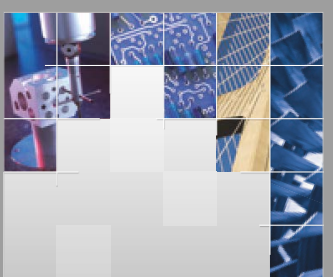

\section{Enfincering}
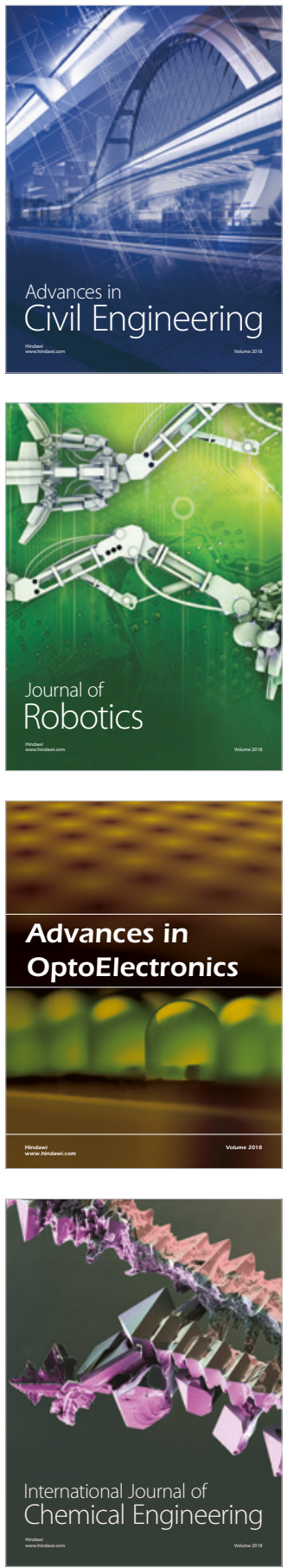

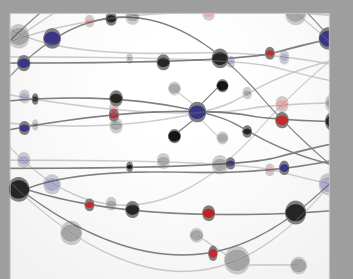

\section{Rotating \\ Machinery}

The Scientific World Journal

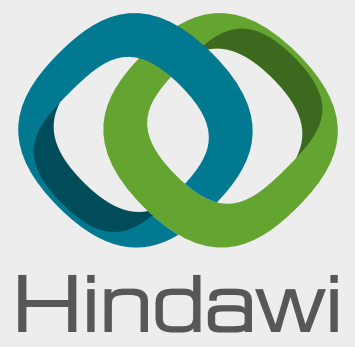

Submit your manuscripts at

www.hindawi.com
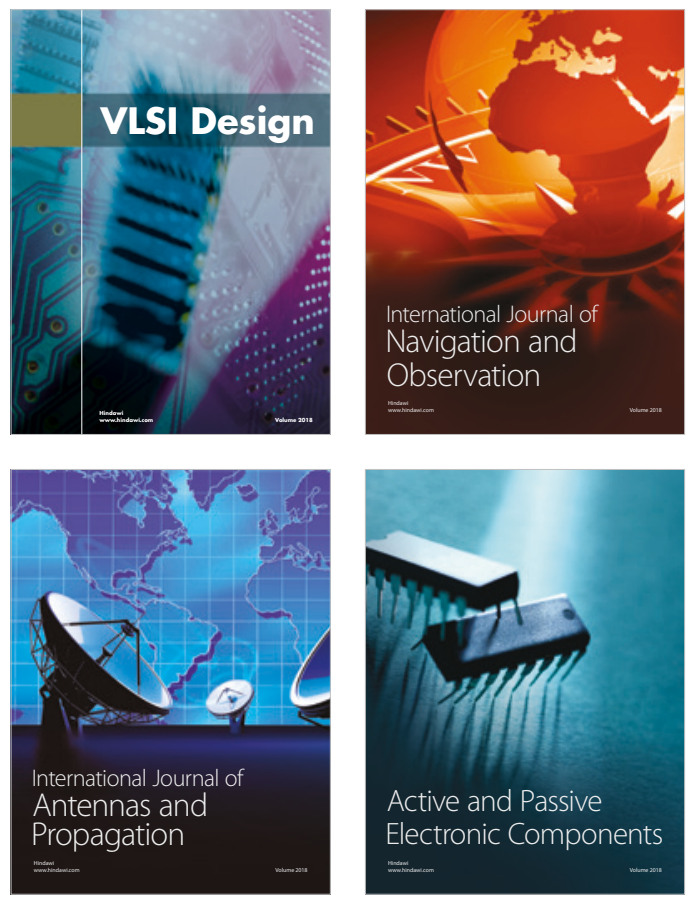
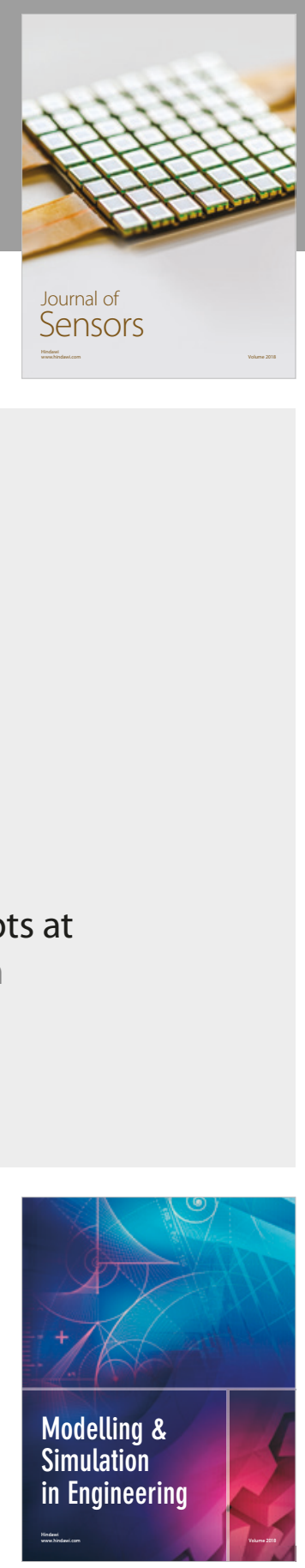

\section{Advances \\ Multimedia}
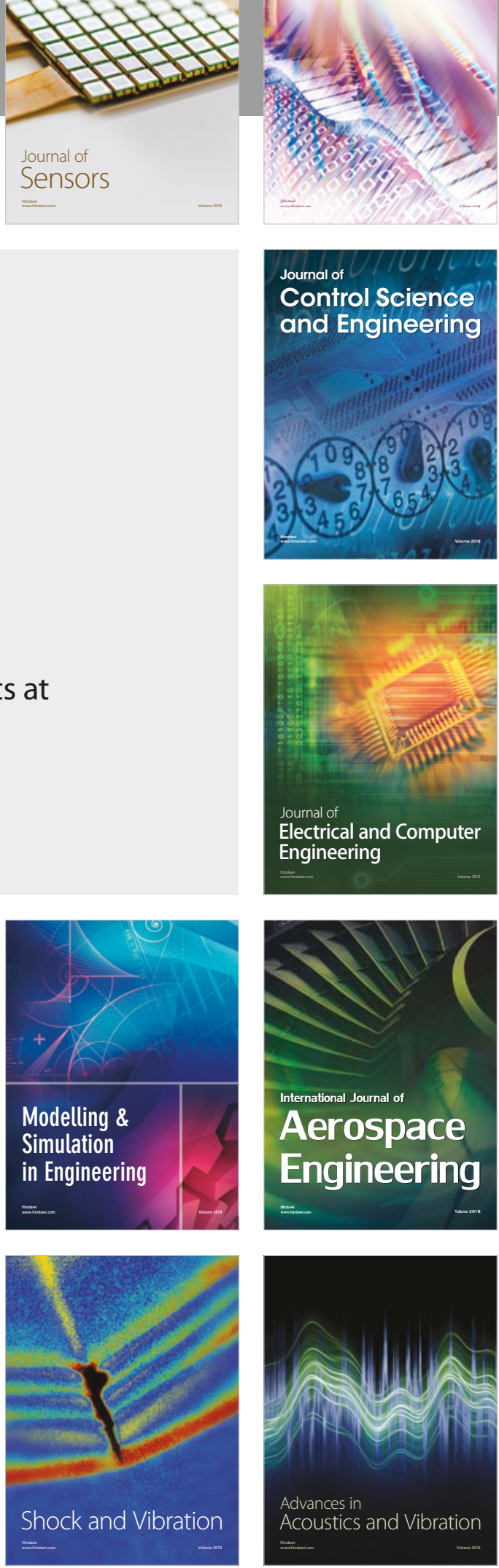